\title{
Tailoring Customer Order Scheduling Search Algorithms
}

\author{
Vahid Riahi ${ }^{\mathrm{a}, *}$, M A Hakim Newton ${ }^{\mathrm{a}}$, M M A Polash ${ }^{\mathrm{b}}$, Abdul Sattar ${ }^{\mathrm{a}}$ \\ ${ }^{a}$ Institute for Integrated and Intelligent Systems (IIIS), Griffith University, Australia \\ ${ }^{b}$ Department of Computer Science and Engineering, Jagannath University, Bangladesh
}

\begin{abstract}
Customer Order Scheduling Problem (COSP) with minimisation of the total completion time as the objective is NP-Hard. COSP has many applications that include the pharmaceutical and the paper industries. However, most existing COSP algorithms struggle to find very good solutions in large-sized problems. One key reason behind is that those algorithms are based on generic templates and as such lack problem specific structural knowledge. In this paper, we capture such knowledge in the form of heuristics and then embed those heuristics within constructive and perturbative search algorithms. In the proposed deterministic constructive search algorithm, we use processing times in various ways to obtain initial dispatching sequences that are later used in prioritising customer orders during search. We also augment the construction process with solution exploration. In the proposed stochastic perturbative search, we intensify its diversification phase by prioritising rescheduling of customer orders that are affected badly. Our tailoring of the search in this case is to make informed decisions when the search has lost its direction. On the contrary to that, in the intensification phase, we then take diversifying measures and use multiple neighbourhood operators randomly so that the search does not get stuck very quickly. Our experimental results show that the proposed algorithms outperform existing state-of-the-art COSP algorithms.
\end{abstract}

Keywords: Customer Order, Scheduling, Heuristic, Local Search

\section{Introduction}

In this paper, we study customer order scheduling problems (COSP). A COSP has $n$ customer orders and $m$ dedicated parallel machines. Each customer order comprises $m$ jobs. Each job can be processed by only a particular machine. A machine can at any time process at most one customer order, but a customer order can be simultaneously processed by more than one machine. Moreover, a machine can start processing another customer order as soon as it completes one. The main goal in COSP is to find a permutation of customer orders so that the total completion time (TCT) is minimised. COSP with minimisation of TCT is NP-Hard (Roemer and Ahmadi, 1997).

COSP has several real-life applications that include car repair shops (Yang, 1998), pharmaceutical industry (Leung et al., 2005a), paper industry (Leung et al., 2005b), and manufacturing of semi finished lenses (Ahmadi et al., 2005). Consider a car repairing shop having several mechanics. Each mechanic can do only a particular type of repairing. However, each arriving car needs several types of repairing. So several mechanics work on each car simultaneously to reduce the completion time for the car. As soon as a mechanic finishes his task on a car, s/he can start working on the next car. A similar model can be considered for ship repairing or aircraft maintenance shops (Yang, 1998). Despite existence of these realistic applications, COSP has not been studied much and so not much progress has been achieved.

*170 Kessels Rd, Nathan QLD 4111

Email addresses: vahid.riahi@griffithuni.edu.au (Vahid Riahi), mahakim.newton@griffith.edu.au (M A Hakim Newton), polash@cse.jnu.ac.bd ( M M A Polash), a.sattar@griffith.edu.au (Abdul Sattar) 
Since COSP with TCT minimisation is NP-Hard, incomplete algorithms have been developed in the literature using heuristics and metaheuristics. One of the best existing heuristics is Earliest Completion Time (ECT) (Leung et al., 2005b). Starting from an empty sequence, at each iteration ECT appends an unscheduled customer order to the already scheduled partial sequence such that TCT of the new partial sequence is the lowest. The time complexity of ECT is $\mathcal{O}\left(m n^{2}\right)$. A very recent heuristic FP (named after the authors' names) is different from ECT in that TCT is computed for the entire sequence assuming unscheduled customer orders as the trailing ones in the sequence (Framinan and Perez-Gonzalez, 2017). The time complexity of FP is $\mathcal{O}\left(m n^{2}\right)$. One possible criticism to both ECT and FP is that they consider placing an unscheduled customer order only at the end of the scheduled partial sequence while other positions between the scheduled jobs could also be considered. Among the metaheuristic algorithms, a tabu search (TS) (Leung et al., 2005b) starts from an ECT solution and iteratively improves further by using swap operators. Moreover, a greedy search algorithm (GSA) (Framinan and Perez-Gonzalez, 2017) starts from an FP solution and performs diversification and intensification repeatedly in an interleaving fashion. In the intensification, GSA explores an exhaustive swap neighbourhood while in the diversification it removes a random customer order, appends it at the end, and then considers inserting each of the other customer orders immediately before it.

Besides the above-mentioned algorithms which are fully proposed for COSP with TCT minimisation, there are some other studies, which focused on other COSP variants. Lin and Kononov (2007) proposed an LP-based approximation algorithm for COSP with minimisation of the number of late jobs as objective. Xu et al. (2015) studied COSP with unrelated parallel machines where each job can be splitted into smaller jobs to be processed by multiple machines. Xu et al. (2016a) studied COSP with minimisation of total tardiness and consideration of learning effects. Lin et al. (2017) proposed a branch-and-bound algorithm as well as particle swarm optimisation for twoagent multi-facility COSP with varying ready times. Wu et al. (2018) considered two objectives, total flowtime and maximum tardiness, for COSP and proposed a hybrid iterated greedy algorithm and a particle swarm colony algorithm in order to solve it. Framinan and Perez-Gonzalez (2018) proposed a heuristic and a metaheuristic for COSP with total tardiness minimisation. Shi et al. (2018) proposed heuristic algorithms in order to minimise the order makespan and total weighted order completion time for COSP on batch processing machines with incompatible job families. Xu et al. (2016b) studied a dynamic COSP with long-run expected order cycle time minimisation as an objective function. Shi et al. (2017) studied COSP with minimising total weighted completion time, and proposed a quadratic formulation for it. Very recently, Framinan et al. (2018) reviewed the assembly scheduling problems and showed that the COSP is a special case of assembly scheduling problems.

Although there are several COSP algorithms for TCT minimisation as discussed above, most of these algorithms struggle to find very good solutions in large-sized problems. One key reason behind is that those algorithms are based on generic templates and as such lack problem specific structural knowledge. In this paper, we capture such knowledge in the form of heuristics and then embed those heuristics within constructive and perturbative search algorithms. Our proposed constructive search algorithm uses customer order processing times in various ways to obtain initial dispatching sequences that are later used in a deterministic search procedure to prioritise customer orders. We also augment the solution construction process with solution exploration phases. Our proposed perturbative search algorithm intensifies its diversification phase by prioritising rescheduling of customer orders that are affected badly. For this, we define a measure of extra time a customer order stays under processing. Our tailoring of the search in this case is to make informed decisions when the search has lost its direction. On the contrary to that, in the intensification phase, we then take diversifying measures and use multiple neighbourhood operators randomly so that the search does not get stuck very quickly. Our experimental results show that the proposed algorithms outperform existing state-of-the-art COSP algorithms on 720 benchmark large instances (Framinan and Perez-Gonzalez, 2017). Moreover, in almost all of them our perturbative search algorithm finds 
the new best solutions.

In the rest of the paper, Section 2 formally defines the problem, Sections 3 and 4 describe the proposed constructive and perturbative algorithms, Section 5 presents the experimental results, and Section 6 concludes the paper.

\section{Problem definition}

The COSP with TCT minimisation is formally formulated as follows. It has $n$ different customer orders and $m$ machines arranged in a parallel layout. Each customer order comprises $m$ jobs (product types) in which each job can be processed on one dedicated machine without interruptions or preemptions. Let $p_{i j}$ denote the processing time of $i$ th job of customer order $j$ to be processed on machine $i$. A machine can at any time process at most one customer order. Moreover, a machine can start processing another customer order as soon as it completes one. The aim is to find a permutation of customer orders such that the total completion time TCT is minimised.

Leung et al. (2005b) proved that there is always an optimal solution where all machines process the orders in the same sequence. So, a COSP solution is represented by a permutation $\pi$ of the customer orders indicated the sequence of the orders are executed. Let $[k]$ denote the customer order at the $k$ th position in $\pi$. Also, let the completion time point of $i$ th job of customer order $[k]$ at machine $i$ is calculated as $C_{i[k]}=C_{i[k-1]}+p_{i[k]}$ where $C_{i[0]}=0$. So the completion time point of a customer order $[k]$ is $C_{[k]}=\max _{i=1}^{m} C_{i[k]}$ and the total completion time of a solution $\pi$ is $\operatorname{TCT}(\pi)=\sum_{i=k}^{n} C_{[k]}$. Computing $\operatorname{TCT}(\pi)$ from scratch clearly needs $\mathcal{O}(m n)$ time. Figure 1 depicts an example COSP with 4 customer orders and 3 machines. Nevertheless, the COSP is to find a permutation $\pi$ of the customer orders such that their total completion time is minimised.

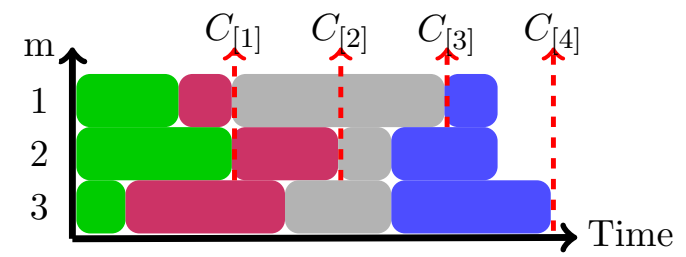

Figure 1: An example COSP with 4 customer orders and 3 machines

\section{Proposed Constructive Heuristic Algorithm}

As discussed before, existing constructive heuristic algorithms such as ECT (Leung et al., 2005b) and FP (Framinan and Perez-Gonzalez, 2017) place an unscheduled customer order only at the end of the already scheduled sequence. In our view, the end of the partial schedule actually might not be the best postition for that unscheduled customer order to be placed, some other earlier positions might be better suited. Those algorithms, however, try each unscheduled customer order and select one of those that results in the best objective value of the new partial sequence.

In our permutation construction and exploration algorithm (PCE) in Algorithm 1, we consider all possible positions in the partial sequence for an unscheduled customer order to be placed in with a view to finding a better position (Lines 3 and 5). Moreover, we consider the unscheduled jobs one by one as they appear in a predefined list $\mathcal{L}$ that is constructed using the processing times of the customer orders (Line 1). The predefined list essentially captures problem instance specific characteristics of a given COSP. We consider 8 ways to create $\mathcal{L}$ and these are described below.

While PCE follows a typical insertion based iterative permutation construction procedure (Lines 5-9), but it then augments the procedure at each iteration by applying swapping based exploration of the same customer order (Line 11-14). The augmented exploration does not increase the over complexity level of the algorithm. The integration of such exploration as part of a constructive method is, we believe, new in the literature, even considering similar other problems. 
Note that the condition $k^{\prime} \notin\left[k_{*}-1, k_{*}+1\right]$ in Line 11 helps avoid revisitation of the permutations created in Lines $5-9$.

To summarise, we make two contributions in our PCE algorithm. As part of the construction process, we find the best position for the best unscheduled customer order in the scheduled partial sequence. Also, we augment the construction process with an exploration procedure.

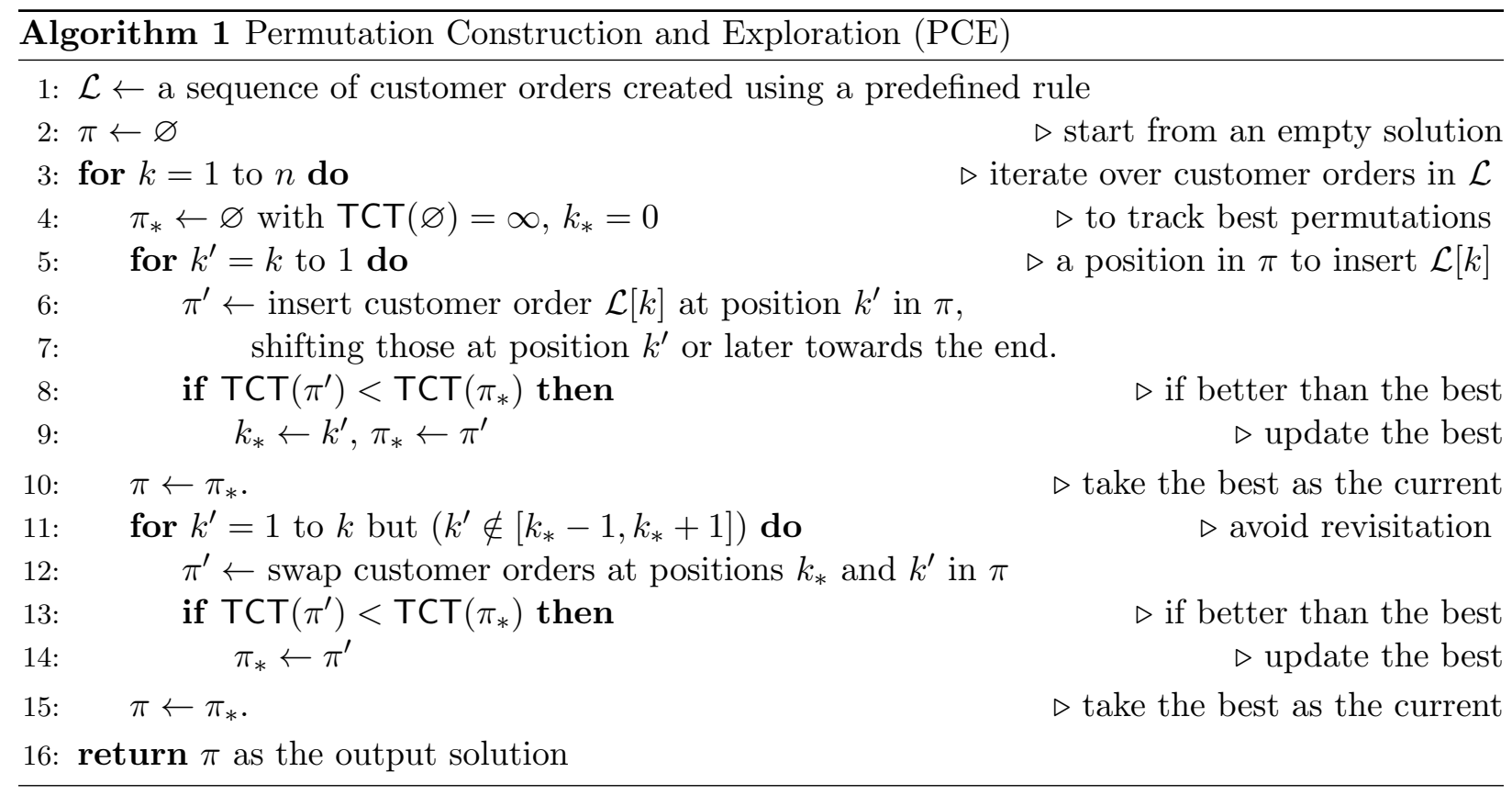

Figure 2 shows an example of insertion based construction followed by swap based exploration in PCE; which is just one lap of the loop in Line 3.

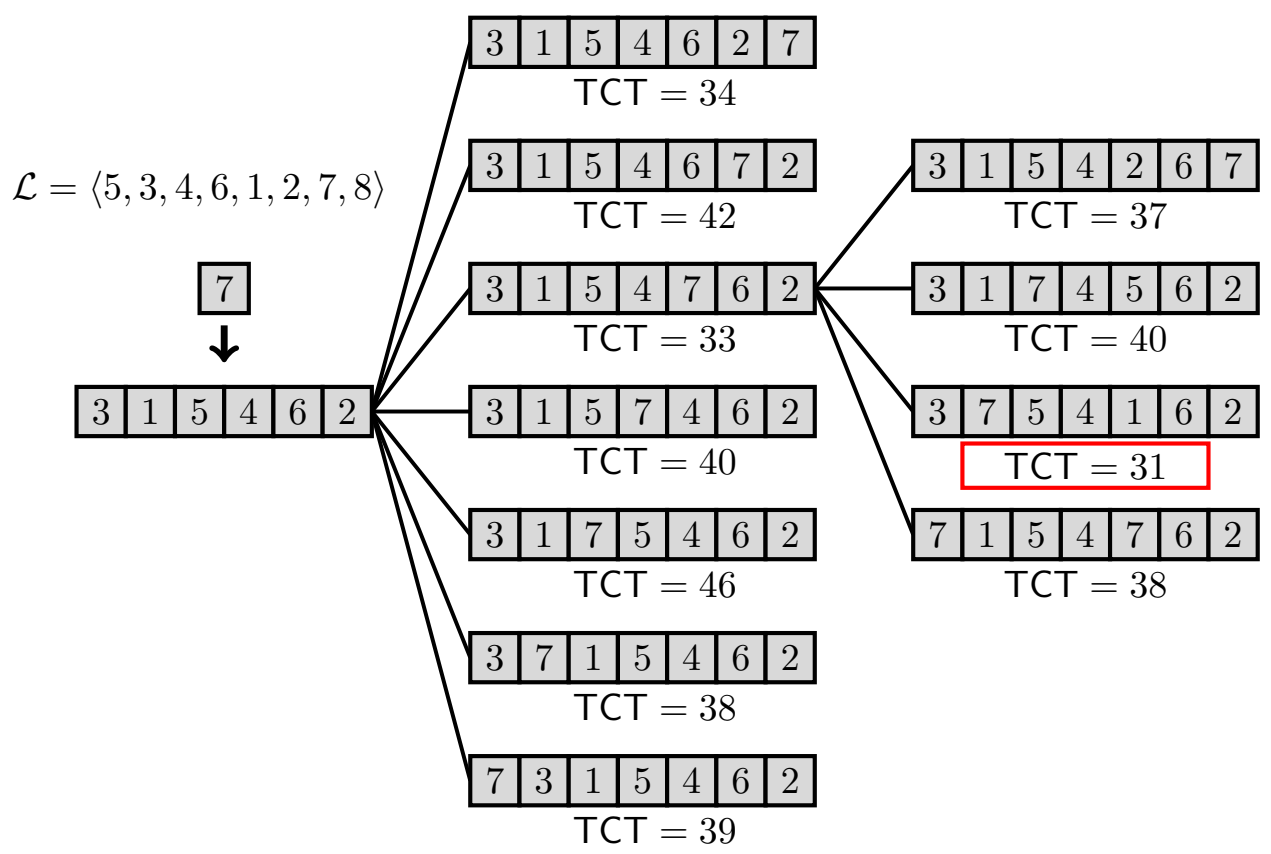

Figure 2: Construction by insertion and exploration by swap in PCE; TCTs are nominal.

Constructing List $\mathcal{L}$. We propose the following 8 ways to construct $\mathcal{L}$. The rough idea is to put a customer order early in $\mathcal{L}$ if it has "short" processing times or has "less" variations in its processing times. This is because an earlier customer order in the solution has an cumulative effect 
on a later one, since total completion time of all customer orders is the objective. The proposed rules below quantify the abstract measures in various ways.

- Machine with the Highest Total Processing Time (HM): Find the machine $i^{\prime}$ such that $i^{\prime}=\operatorname{argmax}_{i} \sum_{j=1}^{n} p_{i j}$. Such a machine determines the makespan of any schedule for a given COSP. Arrange the customer orders in the non-decreasing arrangement on their processing times $p_{i^{\prime} j}$ at machine $i^{\prime}$ to obtain $\mathcal{L}$; the shorter the earlier. The time complexity of using this rule is $\mathcal{O}(n m+n \log n)$.

- Machine with the Lowest Total Processing Time (LM): Find the machine $i^{\prime}$ such that $i^{\prime}=\operatorname{argmin}_{i} \sum_{j=1}^{n} p_{i j}$. Such a machine determines the minimum time period when each machine is busy; after that, some machines would be idle. Arrange the customer orders in the non-decreasing arrangement on their processing times $p_{i^{\prime} j}$ at machine $i^{\prime}$ to obtain $\mathcal{L}$; the shorter the earlier. The time complexity of using this rule is $\mathcal{O}(n m+n \log n)$.

- Machine with the Lowest Total Completion Times (LC): For each machine $i$, obtain a list $\mathcal{L}_{i}$ of customer orders from their non-decreasing arrangement on the processing times $p_{i j}$ on machine $i$. Then, take $\mathcal{L}_{i}$ as $\mathcal{L}$ where $\operatorname{TCT}\left(\mathcal{L}_{i}\right)$ is the minimum. The time complexity of using this rule is $\mathcal{O}\left(n m^{2}+m n \log n\right)$. This rule is used by Framinan and Perez-Gonzalez (2017).

- Machine with the Lowest Weighted Total Completion Time (WLC): This rule is a variant of LC. The only difference is $\mathcal{L}_{i}$ for each $i$ is obtained by arranging customer orders on non-decreasing order of $w_{j} p_{i j}$ instead of just $p_{i j}$ where $w_{j}=\sum_{i=1}^{m} p_{i j} / \sum_{j=1}^{n} \sum_{i=1}^{m} p_{i j}$. The intuition behind this rule is to put the same level of emphasis (i.e. $w_{j}$ ) on a customer order on each machine and the emphasis is based on the relative total processing time of the customer order with respect to the total for all customer orders. The time complexity of using this rules is the same as that of using LC.

- Standard Deviation of Processing Times (SD): In this rule, for each customer order $j$, the standard deviation $\sigma$ of the processing times is calculated from $\sigma_{j}^{2}=\sum_{i=1}^{m}\left(p_{i j}-\right.$ $\left.\mu_{j}\right)^{2} /(m-1)$, where mean $\mu_{j}=\left(\sum_{i=1}^{m} p_{i j}\right) / m$. Then, the customer orders are sorted in a non-decreasing order of the $\sigma_{j}$. The intuition is to give priority to the customer orders with less deviation in the processing times. Such customer orders early will not cause much dispersion of the processing times of the same customer order over all the machines. Thus the time period when a customer order gets processing from at least one machine is reduced. The time complexity of using this rule is $\mathcal{O}(n m+n \log n)$.

- Total Processing Times (TPT): For each customer order $j$, compute the total processing time $\mathrm{TPT}_{j}=\sum_{i=1}^{m} p_{i j}$ over all machines, and then sort the customer orders in the nondecreasing arrangement of $\mathrm{TPT}_{j}$. The time complexity of using this rule is $\mathcal{O}(n m+n \log n)$. This rule is used by Sung and Yoon (1998) to construct a solution directly while we use it just to obtain a predefined list that determines which customer order is inserted and swapped in the each iteration of PCE.

- Total Weighted Processing Times (TWPT): This rule is a variant of TPT. The only difference is customer orders are sorted on the non-decreasing order of $\mathrm{TWPT}_{j}=\sum_{i=1}^{m} p_{i j} \times w_{i}$ instead of $\mathrm{TPT}_{j}=\sum_{i=1}^{m} p_{i j}$ where $w_{i}=\sum_{j=1}^{n} p_{i j} / \sum_{j=1}^{n} \sum_{i=1}^{m} p_{i j}$. The intuition behind this rule is that some machines are more important than others in terms of their relative total processing times measured in $w_{i}$. The time complexity of using this rules is the same as that of using TWPT.

- Total Completion Weighted Processing Time (TCWPT): This rule is a variant of TWPT. The only difference is $w_{i}$ is calculated in a different way. For each machine $i$, obtain 
a list $\mathcal{L}_{i}$ of customer orders from their non-decreasing arrangement on the processing times $p_{i j} \mathrm{~s}$ on machine $i$. Then, for each machine $i$, compute $w_{i}=\operatorname{TCT}\left(\mathcal{L}_{i}\right) / \sum_{i=1}^{m} \operatorname{TCT}\left(\mathcal{L}_{i}\right)$. The time complexity of using this rule is $\mathcal{O}\left(n m^{2}+\right.$ mnlogn $)$.

Below we analyse the time complexity of our proposed PCE algorithm.

Lemma 1. The PCE shown in Algorithm 1 has a time complexity of $\mathcal{O}\left(n^{3} m\right)$.

Proof: Obtaining list $\mathcal{L}$ in the worst case needs $\mathcal{O}\left(n m^{2}+\right.$ mnlogn $)$ time. The nested two level loops and TCT computation altogether needs $\mathcal{O}\left(n^{3} m\right)$ time. So the overall complexity is $\mathcal{O}\left(n^{3} m\right)$.

\section{Proposed Perturbative Search Algorithm}

As mentioned before, existing COSP algorithms struggle to find very good solutions in largesized problems. One key reason is these algorithms are based on generic templates and as such lack problem specific structural knowledge. As a result, they either go for random decisions or for exhaustive neighbourhood exploration. Fully random decisions normally lead to poor performance. On the other hand, exhaustive neighbourhood exploration is very costly and very greedy in nature. These lead to wastage of search effort and premature search convergence. Overall, maintaining diversity level is in general a key issue in a pertubative search. Our proposed perturbative search algorithm intensifies its diversification phase and diversifies its intensification phase. We define a measure of extra time a customer order stays under processing and use this measure in the diversification phase to select customer orders that should be rescheduled. By this, we try to make informed decisions when the search has lost its direction in a local minimum or in a plateau. In the intensification phase, we use multiple neighbourhood operators randomly so the search can get out of the local minima for one neighbourhood operator using another neighbourhood operator as an escape route.

We apply our new strategies on top of a typical perturbative search algorithm (PSA) shown in Algorithm 2. PSA is a single solution iterative search algorithm that explores the solution space. It has four key elements: initialisation, intensification, diversification, and acceptance. PSA starts from an initial solution, in this case, from the solution generated by our constructive heuristic PCE. The initial solution is then improved by using an intensification method. Then, within a loop, the current solution undergoes a diversification method and then the same intensification method in an interleaving fashion. The resultant solution in each iteration replaces the current solution, if the acceptance method accepts it. We describe our intensification, diversification, and acceptance methods in details. We also describe the differences between the proposed PSA and the existing state-of-the-art GSA.

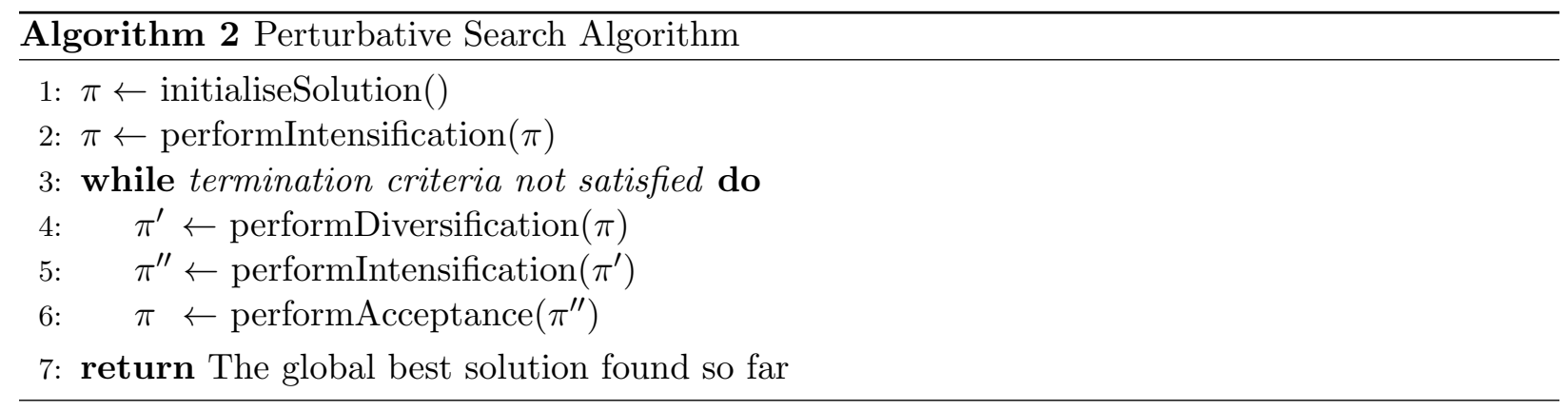




\subsection{Intensification Method}

Algorithm 3 describes our proposed performIntensification() method. It starts from an input solution $\pi$. For a given number of iterations $N$, it then applies a randomly selected neighbourhood operator from a set of such operators $\mathcal{N}$. If the result solution $\pi^{\prime}$ is better than the current solution $\pi$ in terms of TCT, then $\pi^{\prime}$ replaces $\pi$. We use one of the operators at a time from $\mathcal{N}$. This is because applying all of them every time is very time costly. Also, using one at a time helps the search get out of local optima of another one used before. In fact this is the reason, we use multiple neighbourhood operators in this way to diversify the intensification phase.

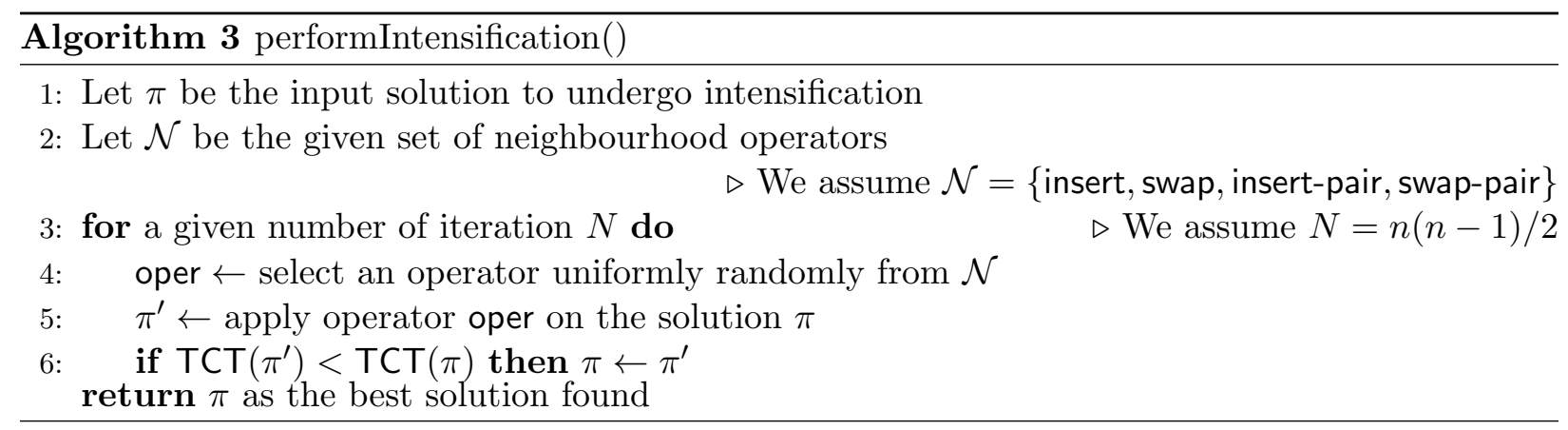

In this paper, we use the following four neighbourhood operators. These operators are common in problems where the solutions can be represented by permutations, e.g. in flowshops (Riahi et al., 2018).

insert $\left(\pi, k, k^{\prime}\right)$ : the customer order at position $k$ in $\pi$ is at position $k^{\prime}$ in the resultant permutation $\pi^{\prime}$. If $k>k^{\prime}$, any customer orders at positions $k^{\prime} \leq k^{\prime \prime}<k$ move to positions $k^{\prime \prime}+1$. If $k<k^{\prime}$, any customer orders at positions $k<k^{\prime \prime} \leq k$ move to positions $k^{\prime \prime}-1$.

$\operatorname{swap}\left(\pi, k, k^{\prime}\right)$ : swap two customer orders at positions $k$ and $k^{\prime}$ in $\pi$ to obtain the resultant permutation $\pi^{\prime}$.

insert-pair $\left(k, k^{\prime}\right)$ : two customer orders at positions $k$ and $k+1$ are respectively at positions $k^{\prime}$ and $k^{\prime}+1$ in the resultant permutation $\pi^{\prime}$ where $\left|k-k^{\prime}\right| \geq 2$. If $k>k^{\prime}$, any customer orders at positions $k^{\prime} \leq k^{\prime \prime}<k$ move to positions $k^{\prime \prime}+2$. If $k<k^{\prime}$, any customer orders at positions $k+1<k^{\prime \prime} \leq k^{\prime}+1$ move to positions $k^{\prime \prime}-2$.

swap-pair $\left(k, k^{\prime}\right)$ : swap two customer orders at positions $k+1$ and $k^{\prime}+1$ in $\pi$ along with swapping those at positions $k$ and $k^{\prime}$ to obtain the resultant permutation $\pi$ where $\left|k-k^{\prime}\right| \geq 2$.

Whenever one of the four operators selected we also uniformly randomly select positions for the parameters $k$ and $k^{\prime}$. For the number iterations, we set $N=n(n-1) / 2$ since this value is equal to the number of possible swaps in an exhaustive swap based neighbourhood operator and is used by the state-of-the-art GSA algorithm (Framinan and Perez-Gonzalez, 2017). We later experimentally compare our proposed PSA with GSA.

Given all four operators described above, customer orders at positions before $k^{\prime \prime}=\min \left(k, k^{\prime}\right)$ remain the same before and after the application of a selected operator. So in our implementation, while calculating the total completion time for the resultant permutation, we reuse the completion times of these customer orders and recompute only for those after position $k^{\prime \prime}$.

\subsection{Diversification Method}

Algorithm 4 describes our proposed performDiversification() method. Assuming $D$ is a parameter to be chosen by experiments, the proposed diversification method removes $D$ selected customer orders from the input solution $\pi$ (Line 5). We discuss selection of the $D$ customer orders later, but $\mathcal{L}$ is the list to hold them. Given $\mathcal{L}$ the list of customer orders removed and $\pi$ the resultant 
solution after removal, each of the customer order in $\mathcal{L}$ is then inserted back to $\pi$ and also swapped with other customer orders already in $\pi$ (Lines 7-17). Note that Lines 7-17 in Algorithm 4 are the same as Lines 4-14 in Algorithm 1. As such we do not describe them further, but do reiterate that a swapping based exploration is an important augmentation to the insertion based construction typically seen in similar methods.

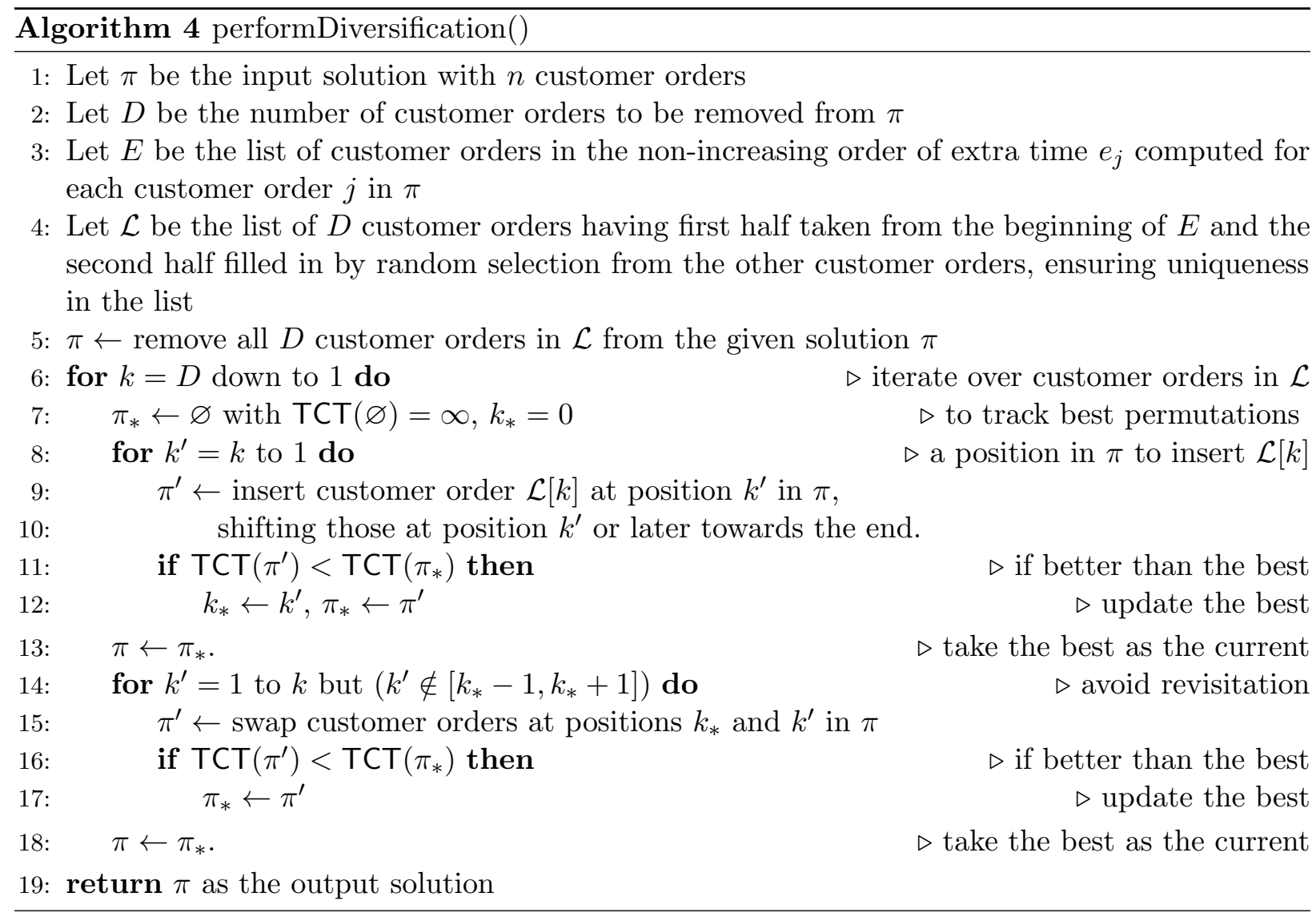

Our key contribution in the proposed diversification method is mainly in the selection of the $D$ customer orders to be removed and inserted back to the given solution. Typically only random selections are used in such scenarios. Note that the diversification method is called after the intensification phase, which perhaps have already taken the search to a local minimum or a plateau. The search has therefore lost its direction. Making only random decisions at that point appears to be relying only on luck while an intelligent search approach would rather make informed decisions.

In this paper, we try to identify the customer orders that are very badly affected in a given solution $\pi$. We prefer to reschedule them which can be viewed as fixing the problematic part of a solution. For this, we define a measure of the extra time $e_{j}$ a customer order $j$ stays under processing in a given solution $\pi$. A customer order $j$ should ideally be staying under processing for a time window with length at most $\eta_{[k]}=\max _{i=1}^{m} p_{i j}$, which is the longest processing time of $j$ on any machine. Since a machine can start processing the next customer order as soon as it completes the previous one, the time a customer order $[k]$ in $\pi$ actually remains under processing is given by $\omega_{[k]}=\max _{i=1}^{m} C_{i[k]}-\min _{i=1}^{m} C_{i[k-1]}$. So $e_{[k]}=\omega_{[k]}-\eta_{[k]}$ can be used to measure the extra time customer order $[k]$ stays under processing. In the proposed diversification method, the higher $e_{[k]}$ values are used to select $D / 2$ customer orders to be removed (Lines 3-4 in Algorithm 4). The other $D / 2$ customer orders to be removed are however selected randomly.

Figure 3 shows computation of the extra times 4 customer orders in an example COSP solution with 4 machines. Notice that $e_{[1]}=4-0-4=0, e_{[2]}=8-2-5=1, e_{[3]}=11-5-3=3$, $e_{[4]}=13-7-4=2$. 


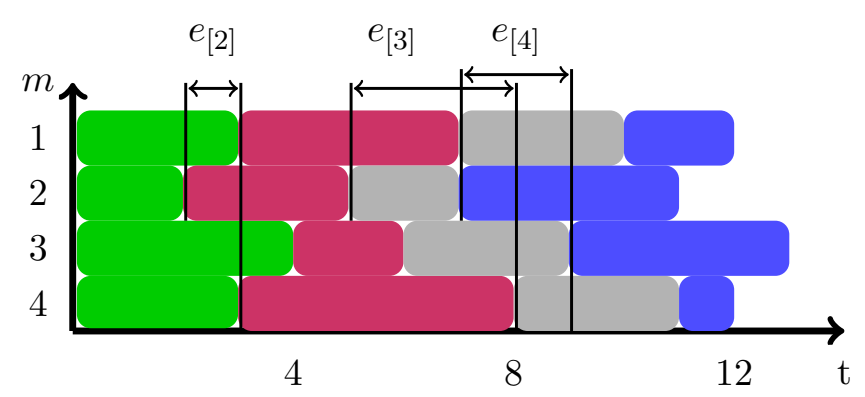

Figure 3: An example of computing extra time for each customer order

\subsection{Acceptance Method}

For the acceptance method, we consider $\Delta_{0}$ that is the difference in the TCT obtained by the search procedure (lines 3-15 of Algorithm 1) of the PCE algorithm. Then, given a parameter $R$ to be experimentally determined, we define a threshold $R \times \Delta_{0}$. Now, in each iteration of Algorithm 2, $\pi$ is the solution before diversification and $\pi^{\prime \prime}$ is the solution after intensification. We accept $\pi^{\prime \prime}$ to be next current solution $\pi$ if $\operatorname{TCT}\left(\pi^{\prime \prime}\right)-\operatorname{TCT}(\pi) \leq R \times \Delta_{0}$. Notice that improving solutions are always accepted in this method and worsening solutions are also accepted but only when the differences are within the threshold limit. Notice that the threshold limit depends on each problem size and even problem instances.

\subsection{Overall difference with GSA}

GSA algorithm is the state-of-the-art algorithm for COSP with TCT minimisation. We discuss the main difference of our algorithm from GSA.

In the diversification phase, GSA moves a random customer order to the last position $n$ and then try to find the best customer order for the position $n-1$. If a better solution is found, the process continues. However, PSA uses an informed diversification technique exploiting problem specific knowledge. In order to do that, it calculates a measure of extra time that a customer order stays under processing and uses this measure to select customer orders that should be rescheduled. The effectiveness of the proposed informed diversification is shown in Section 5.4.

Also, in the intensification phase, GSA uses an exhaustive swap move while PSA uses a multi neighbourhood mechanism; both algorithms consider the same number of possible moves. It allows our algorithm to navigate through the search space, to explore more areas around the current solution, and to escape from local optima. The advantages of using multiple neighbourhood against an exhaustive swap move is shown in Section 5.3.

\section{Experimental Results}

We evaluate our algorithms using the benchmark set generated by Framinan and Perez-Gonzalez (2017). However, we only use 720 large instances. These instances are equally divided into two groups: Test- 1 and Test-2. Each group is further divided into $3 \times 4=12$ sets, one for each combination of $n \in\{50,100,200\}$ and $m \in\{2,5,10,20\}$. Each of the 12 sets thus has 30 instances. While Test-2 instances allow zero processing times $p_{i j} \in[0,100)$, Test- 1 instances does not allow that $p_{i j} \in(0,100)$. We also use another set of 90 instances, which are generated by us and are divided into two similar instance groups. For this, we use $n \in\{50,100,200\}$ and $m \in\{5,10,20\}$, and for each combination of $n$ and $m$, we generate 5 instances per group. The processing times have the same distributions as in Test- 1 and Test2. These generated instances are used to calibrate the parameters and components of our algorithms while the benchmark set mentioned before is used in comparing our algorithm with the state-of-the-art algorithms.

We run each deterministic constructive algorithm only once on each instance. We run each stochastic perturbative algorithm on each instance 5 times on the same machine. The termination 
criterion in the proposed PSA algorithm and the GSA algorithm that we compare PSA with is the maximum iteration IterMax. This criterion was used in the evaluation of GSA.

The values used for IterMax in PSA and GSA are mentioned later whenever needed. Given that both algorithms in each iteration explore the same number of neighbouring solutions, and as we practically found, the time taken by the two algorithms are very close for a given value of IterMax. Note that it is not possible to run the GSA executable program provided by the respective author using timeout criterion.

Let $T C T_{a}$ be the TCT value found by a given algorithm and $T C T_{r}$ be the reference TCT value. For each run of each algorithm on each instance, we calculate the relative percentage deviation $\mathrm{RPD}=\frac{\mathrm{TCT}_{\mathrm{a}}-\mathrm{TCT_{ \textrm {r } }}}{\mathrm{TCT}_{\mathrm{r}}} \times 100$ of the total completion time of the schedule found. To summarise further, we calculate average RPD (ARPD) for a given algorithm over the runs of an instance, or over the instances in an instance set or in an instance group.

\subsection{Analysis of Constructive Heuristics}

Using 8 ways to construct an initial priority list $\mathcal{L}$ of customer orders, we obtain 8 variants of the proposed PCE algorithm. For each $\mathcal{L}$, we denote the respective PCE variant by PCE- $\mathcal{L}$. These are compared with FP (Framinan and Perez-Gonzalez, 2017) on the 720 benchmark instances. Table 1 shows in details the ARPDs for each instance set in each instance group for the PCE variants (names without minus signs) and FP. Among all PCE variants, PCE-TPT, PCE-TWPT, and PCE-TCWPT outperform the other PCE variants and FP. Figure 4 shows the ARPDs and also the Tukeys Honest Significant Difference (HSD) confidence intervals. In this figure, we also show the PCE variants without the swap based exploration (names with minus signs). This help us to find out the effect of swap based exploration on the algorithm performance. As can be seen, the swap based explorations improve the effectiveness of the PCE heuristics.

Nevertheless, since our proposed algorithm has a different complexity compared to FP, we also compared the algorithms in terms of CPU times. Figure 5 depicts the ARPDs versus average CPU times for all heuristics. From this figure, we can see that all the proposed constructive heuristics need less than 0.14 second on an average. Considering the average time (shown in Table 5) that we spend to solve each instance by using local search algorithms, 0.14 second is negligible. The FP algorithm has a lower time complexity and consequently it is faster than all PCE variants, but the ARPD of FP is better than that of most PCE variants. The use of swap-based exploration increase the time required by the PCE variants but achieves significantly better ARPDs.

\subsection{Parameter Tuning for PSA}

The proposed PSA has only two parameters that are to be carefully calibrated. These parameters are $D$ the total number of customer orders to be removed in the diversification phase and $R$ the threshold determining ratio to be used in the acceptance method. We consider $D \in\{2,3,4,5,6\}$ and $R \in\{0.01,0.001,0.05,0.005\}$ after preliminary experiments. We then use the Design of Experiments method (Montgomery, 2017) to find the best value for each parameter. For this, we use the 90 generated instances mentioned before and use IterMax $=200$, and also TWPT as the initial solution. In computing the ARPDs, we use the best TCT found by any of the versions run in this calibration process.

Table 2: ANOVA results for the two parameters of PSA.

\begin{tabular}{cccccc}
\hline Source & Degrees of freedom & Sum of squares & Adjusted mean square & F-statistic & P-value \\
\hline \hline$D$ & 4 & 43.119 & 10.7798 & 1127.01 & $\mathbf{0 . 0 0 0}$ \\
$R$ & 3 & 1.293 & 0.4312 & 45.08 & $\mathbf{0 . 0 0 0}$ \\
$R \times D$ & 12 & 0.601 & 0.0501 & 5.24 & $\mathbf{0 . 0 0 0}$ \\
Error & 8980 & 85.893 & 0.0096 & & \\
Total & 8999 & 130.907 & & & \\
\hline
\end{tabular}

We analyse the results by means of multi-factor analysis of variance (ANOVA) method. The ANOVA results are given in Table 2, where those with the p-value less than 0.05 are highlighted. 
Table 1: Comparison of constructive heuristic algorithms PCE and FP using ARPDs. For each $\mathcal{L} \in$ $\{H M, L M, L C, W L C, S D, T P T, T W P T, T C W P T\}$, there is a PCE variant PCE- $\mathcal{L}$ and column $\mathcal{L}$ shows its ARPDs.

\begin{tabular}{|c|c|c|c|c|c|c|c|c|c|}
\hline \multirow[b]{2}{*}{ Instance } & \multicolumn{8}{|c|}{ PCE variants with various $\mathcal{L}$} & \multirow[b]{2}{*}{$\mathrm{FP}$} \\
\hline & HM & LM & $\mathrm{LC}$ & WLC & SD & TPT & TWPT & TCWH & \\
\hline \multicolumn{10}{|c|}{ TEST-1 } \\
\hline $50 \times 2$ & 2.031 & 2.111 & 2.056 & 0.596 & 2.133 & 0.053 & 0.02 & 0.105 & 0.2 \\
\hline $50 \times 5$ & 184 & 753 & 1.718 & 0.785 & 2.766 & 0.213 & 0.104 & 184 & 47 \\
\hline $50 \times 10$ & 867 & 2.002 & 1.719 & 1.023 & 2.514 & 0.258 & 0.144 & 329 & .493 \\
\hline $50 \times 20$ & 754 & 153 & 532 & 1.038 & 2.137 & 0.405 & 0.279 & 351 & .315 \\
\hline $100 \times 2$ & 2.984 & 85 & 984 & 0.984 & 2.628 & 0.050 & .015 & 990 & .246 \\
\hline 10 & 107 & 16 & 935 & 0.814 & 2.486 & 0.137 & .0 & J & .568 \\
\hline $100 \times 10$ & 1.936 & 4 & 665 & 0.954 & 2.339 & 0.162 & .071 & 148 & .540 \\
\hline $100 \times 20$ & 1.828 & 19 & 765 & 1.243 & 2.209 & 0.203 & 1.1 & 186 & .308 \\
\hline 20 & 3.344 & 0 & 3.344 & 1. & 2.484 & 0.043 & 0.005 & 5 & .200 \\
\hline 20 & 2.342 & 1.705 & 116 & 0.8 & 2.496 & 0.072 & - & 014 & .841 \\
\hline 10 & 838 & 701 & 592 & 1. & 2.352 & 0.065 & 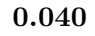 & 5 & .558 \\
\hline $200 \times 20$ & 1.819 & 2.071 & 793 & 1.346 & 2.166 & 0.089 & 0.071 & 185 & 0.362 \\
\hline average & 3.518 & 3.008 & 3.184 & 1.448 & 3.430 & 0.195 & 0.078 & 0.208 & 1.057 \\
\hline \multicolumn{10}{|c|}{ TEST-2 } \\
\hline $50 \times 2$ & 2.883 & 2.939 & 2.863 & 0.976 & 2.902 & 0.104 & 0.026 & 21 & 0.606 \\
\hline $50 \times 5$ & 2.957 & 2.434 & 2.429 & 1.204 & 3.703 & 0.308 & 0.208 & 415 & .924 \\
\hline $50 \times 10$ & 3.279 & 3.083 & 2.614 & 1.459 & 3.480 & 0.548 & 0.175 & 0.475 & 0.843 \\
\hline $50 \times 20$ & 3.274 & 3.614 & 2.671 & 1.923 & 2.807 & 0.510 & 0.311 & 0.407 & 0.897 \\
\hline $100 \times 2$ & 3.567 & 3.554 & 3.565 & 1.097 & 2.777 & 0.063 & 0.009 & 0.150 & 0.574 \\
\hline $100 \times 5$ & 3.740 & 2.496 & 3.169 & 1.278 & 4.021 & 0.277 & 0.038 & .242 & 1.466 \\
\hline $100 \times 10$ & 3.377 & 2.906 & 2.918 & 1.472 & 3.684 & 0.226 & 0.096 & .294 & 1.063 \\
\hline $100 \times 20$ & 2.963 & 3.056 & 2.953 & 1.384 & 2.927 & 0.223 & 0.237 & 0.228 & 0.695 \\
\hline $200 \times 2$ & 4.380 & 4.368 & 4.383 & 1.510 & 3.132 & 0.046 & 0.001 & 111 & 0.471 \\
\hline $200 \times 5$ & 3.648 & 2.154 & 3.070 & 1.245 & 3.822 & 0.155 & 0.010 & 198 & 1.839 \\
\hline $200 \times 10$ & 3.368 & 2.683 & 2.808 & 1.482 & 3.534 & 0.170 & 0.081 & 170 & 1.234 \\
\hline $200 \times 20$ & 3.277 & 3.352 & 3.124 & 1.812 & 3.006 & 0.227 & 0.056 & 0.167 & 0.740 \\
\hline average & 3.393 & 3.053 & 3.047 & 1.403 & 3.316 & 0.238 & 0.104 & 0.256 & 0.94 \\
\hline
\end{tabular}

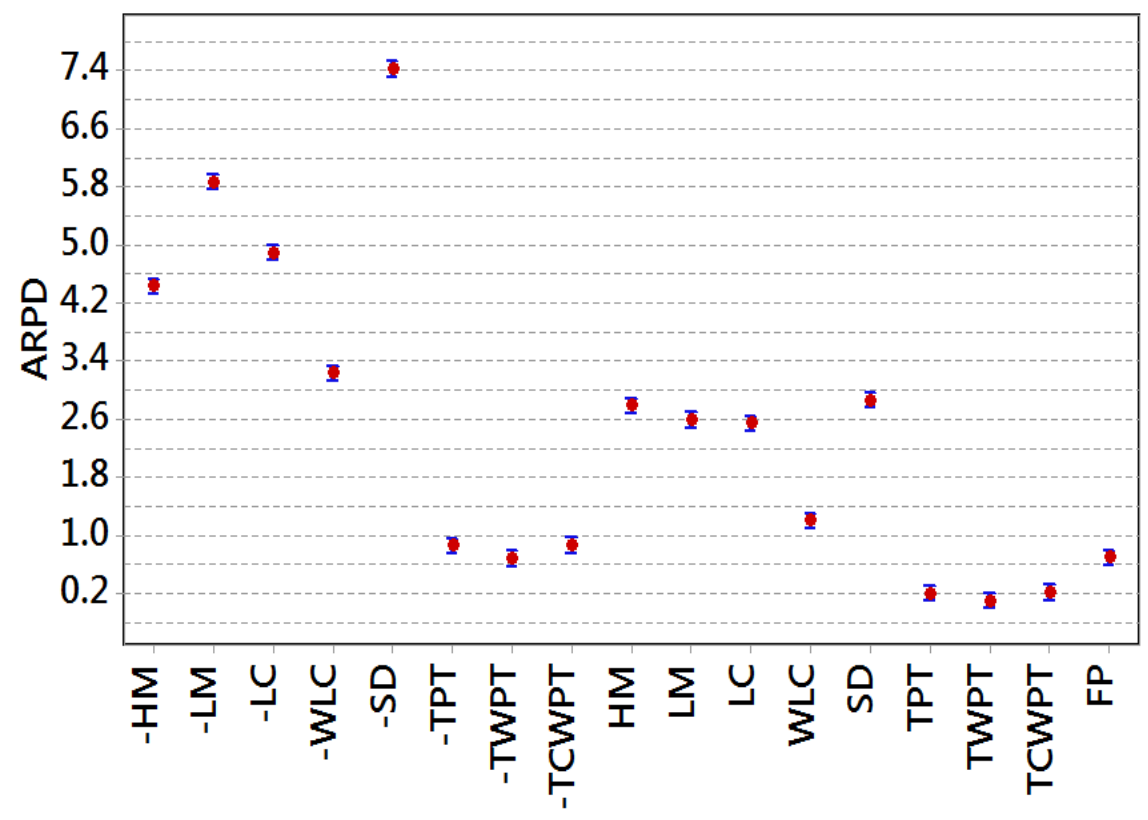

Figure 4: ARPDs and 95\% HSD confidence intervals for PCE variants and FP. Names in x-axis with/without minus signs denote PCE versions without/with swap-based exploration. For each $\mathcal{L} \in\{\mathrm{HM}, \mathrm{LM}, \mathrm{LC}, \mathrm{WLC}, \mathrm{SD}, \mathrm{TPT}$, TWPT, TCWPT $\}$, we use $\mathcal{L}$ when PCE- $\mathcal{L}$ includes swap-based explorations and $-\mathcal{L}$ when does not.

Three main ANOVA hypotheses i.e., normality, homoskedasticity of the different levels, and independence of the residuals are firstly checked and no significant deviations are found. From the 


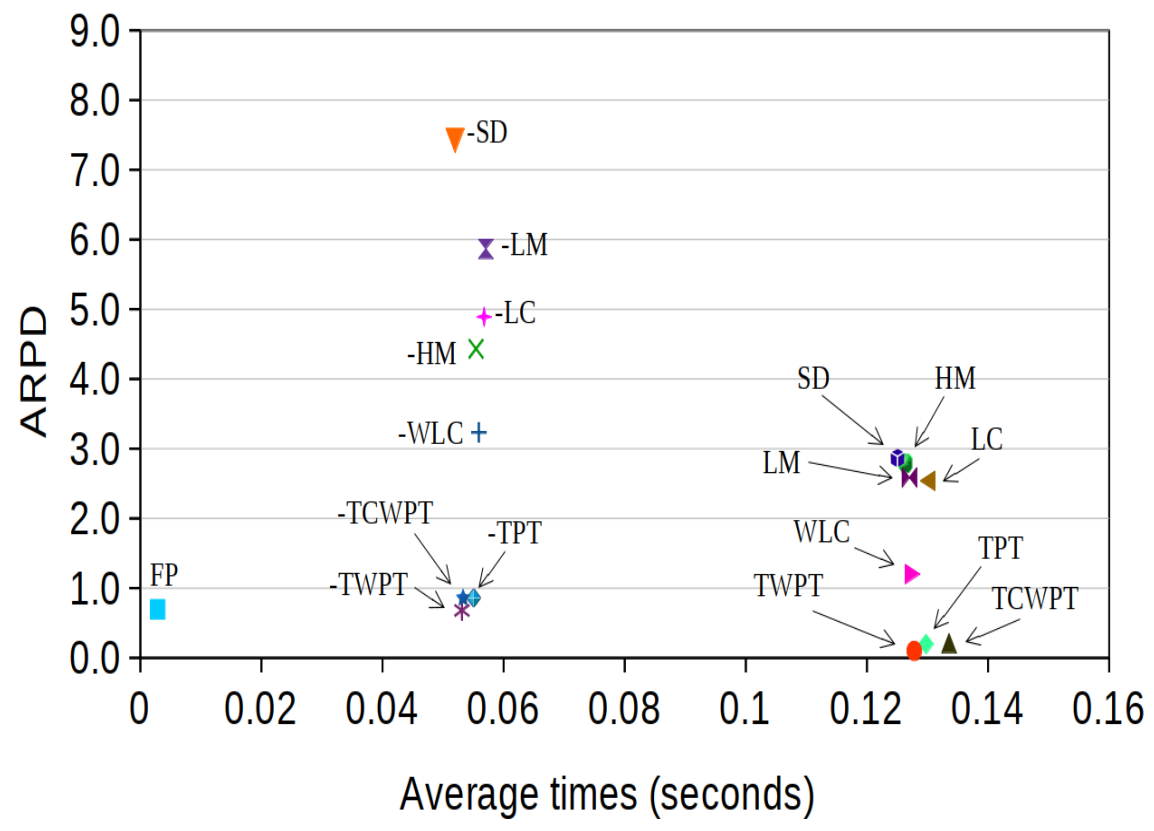

Figure 5: ARPDs versus CPU time for PCE variants and FP. Names with/without minus signs denote PCE versions without/with swap-based exploration. For each $\mathcal{L} \in\{\mathrm{HM}, \mathrm{LM}, \mathrm{LC}, \mathrm{WLC}, \mathrm{SD}, \mathrm{TPT}, \mathrm{TWPT}, \mathrm{TCWPT}\}$, we use $\mathcal{L}$ when PCE- $\mathcal{L}$ includes swap-based explorations and $-\mathcal{L}$ when does not.

table, we can first see that the $D$ is the most significant factor, since it has the highest F-statistic. The p-value of both factors are less than 0.05, which means that there is a significant difference between at least two levels of each factor. To find out the best level for the factors, the $95 \%$ HSD confidence interval plots are shown in Figure 6.
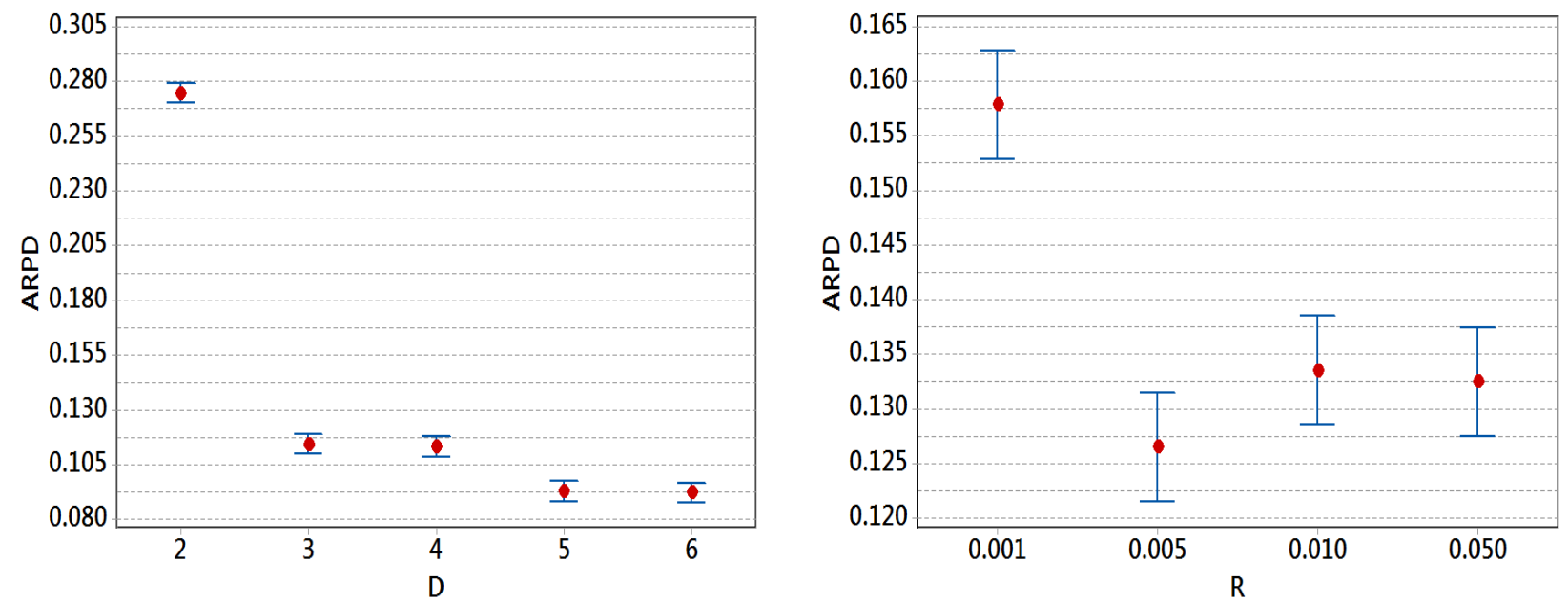

Figure 6: ARPDs and 95\% HSD confidence intervals for parameters $D$ (left) and $R$ (right)

From Figure 6-left, we see that $D=2$ obtained the worst results. Overall, PSA obtained better results with larger $D$ values. There is no statistically significant difference between using $D=5$ and $D=6$, where ARPDs are 0.0931 and 0.0923 respectively. However, we select $D=6$ since it results in a lower ARPD. In terms of parameter $R$, we see in Figure 6-right that $R=0.001$ is the worst case because it rejects most non-improving solutions. Among 0.005, 0.10, and 0.05, we select $R=0.005$ as it gives the lowest ARPD although these three values produce results that are statistically not significantly different. Since the interaction of factors $R \times D$ is significant, we also 
see the interaction plot shown in Figure 7. From this figure, it is clear that the version with $D=6$ and $R=0.005$ produces the lowest ARPD.

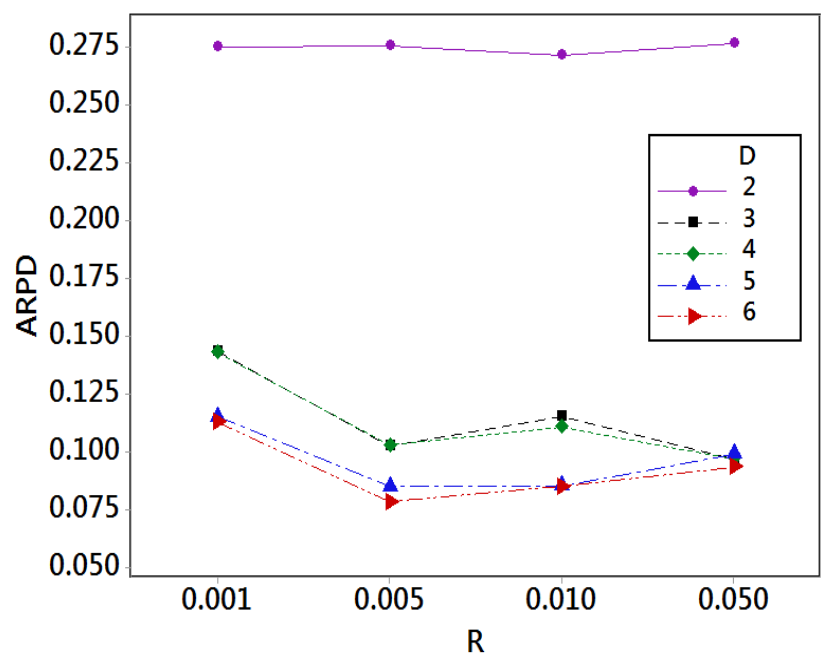

Figure 7: Interaction plot for $D$ and $R$.

\subsection{Analysis of Diversified Intensification}

In the intensification phase, we use four neighbourhood operators: insert (I), swap (S), insert-pair (IP), and swap-pair (SP). We evaluate their impact on the search performance the proposed PSA. For this, we use the 90 generated instances mentioned before, IterMax $=200$, and also TWPT as the initial solution. In computing the ARPDs, we use the best TCT found by any of the versions run in this calibration process. The $95 \%$ HSD confidence interval plot is shown in Figure 8.

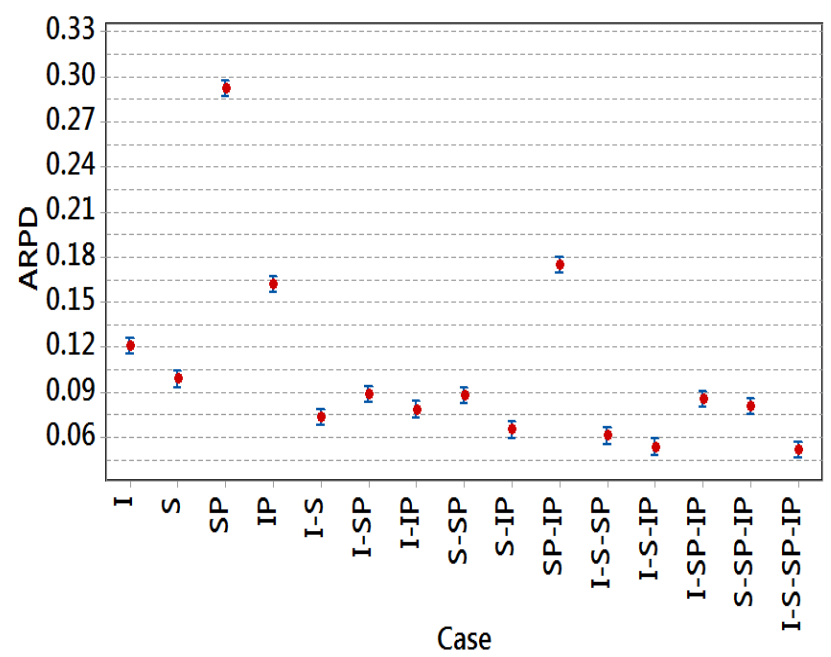

Figure 8: 95\% HSD interval plot for different intensification cases.

From Figure 8, we see that when each operator is used separately, the swap operator obtains the best results while the swap-pair operator obtains the worst. When using two operators at a time, results are significantly better than using single operators and insert, swap $\}$ and \{swap, insert-pair\} appears to be performing the best. When considering three or four operators at a time, using all four operators appears to be the best but not significantly better than using \{insert, swap, insert-pair\}. Therefore, we use all four operators in the final version of our PSA algorithm. 


\subsection{Analysis of Intensified Diversification}

In the diversification phase, we use extra time that a customer order stays under processing to select customer orders that are to be removed from a schedule and inserted back. We compare this greedy strategy (denoted by $\mathrm{G}$ ) with a random selection strategy (denoted by $\mathrm{R}$ ). We also compare the proposed diversification strategies with the one used by GSA algorithm (donated by S). For this, we use the 90 generated instances mentioned before, IterMax $=200$, and also TWPT as the initial solution. In computing the ARPDs, we use the best TCT found by any of three versions run in this calibration process. The 95\% HSD confidence interval plot reported in Figure 9 confirms that our proposed strategy G significantly outperforms the typical random one as well as GSA's diversification method.

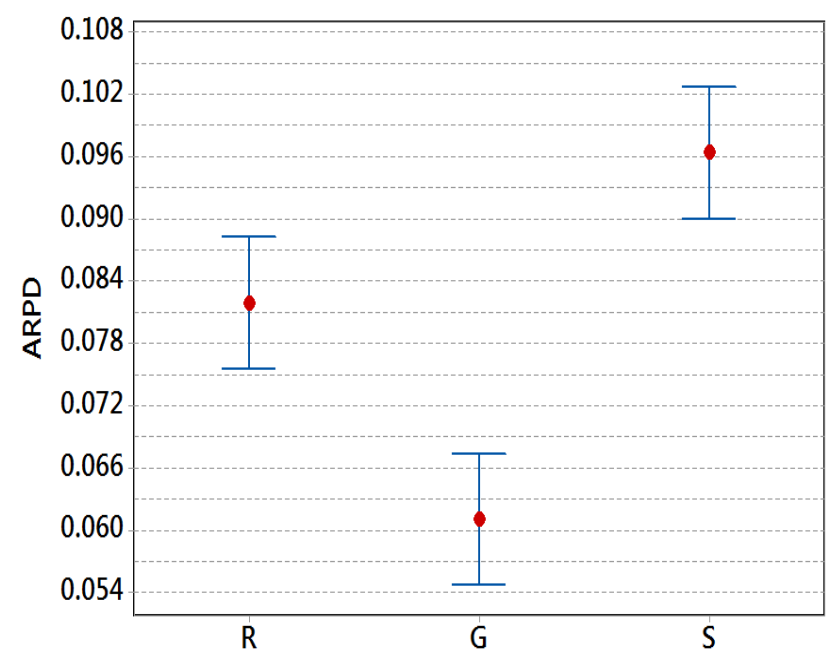

Figure 9: 95\% confidence interval plot of the PSA with different diversification variants.

\subsection{Comparison of PSA with GSA}

We compare the final version of the proposed PSA with the state-of-the-art COSP algorithm GSA (Framinan and Perez-Gonzalez, 2017). For initialisation in PSA, we use our proposed PCE algorithm with TWPT and SD initial sequencing strategies. TWPT and SD respectively obtain the best and the worst performance in PCE. We include SD to see how PSA performs even with the worst initial solution ( $2 \%$ worse than FP produced solutions). We also use FP (Framinan and PerezGonzalez, 2017) within PSA. Proposed algorithms are all implemented in programming language $\mathrm{C}$, while GSA implementation is obtained from the respective authors. In this experiment, we use 720 benchmark instances and six different stopping criteria IterMax $\in\{10,50,100,200,500,1000\}$. Note that, GSA algorithm was tested only for IterMax $\in\{10,50,100\}$. For ARPD calculation, the best solutions found by any algorithm are used as references. The results are shown in Table 3 and Table 4 for Test- 1 and Test-2 instance groups respectively.

From those two tables, we see that all PSA variants obtained ARPDs lower than GSA. Interestingly, PSA obtains similar performance regardless of which initial solution is used. This shows PSA's robustness. Further, ARPDs obtained by PSA variants after 100 iterations are better than those obtained by GSA and after 1000 iterations, become four times better. However, ARPDs improve for all algorithms with the increase of the numbers of iterations. Similar observations can also be made from the 95\% HSD confident intervals for Test-1 and Test-2 in Figure 10 and 11 respectively.

To observe the effectiveness of the proposed PSA further, we also show the convergence of GSA and PSA algorithms in four selected instances-Test-1-347, Test-1-463, Test-2-312, and Test2-469-in Figure 12. Since GSA used FP as the initial solution, we also use it as our starting point 
Table 3: ARPDs for the search algorithms on Test-1

\begin{tabular}{|c|c|c|c|c|c|c|c|c|c|c|c|c|}
\hline & \multicolumn{6}{|c|}{ GSA } & \multicolumn{6}{|c|}{ PSA-FP } \\
\hline Ins. & 10 & 50 & 100 & 200 & 500 & 1000 & 10 & 50 & 100 & 200 & 500 & 1000 \\
\hline $50 \times 2$ & 0.076 & 0.042 & 0.035 & 0.026 & 0.023 & 19 & 0.024 & 0.007 & 0.005 & 0.003 & 0.001 & 0.001 \\
\hline $50 \times 5$ & 306 & 0.192 & 0.147 & 0.132 & .108 & 097 & 175 & 0.079 & 0.057 & 0.040 & 0.024 & 0.016 \\
\hline $50 \times 10$ & 471 & 0.264 & 0.232 & 0.202 & 0.163 & 0.141 & .372 & 0.200 & 0.156 & 0.114 & 0.079 & 0.050 \\
\hline $50 \times 20$ & 536 & 0.316 & 0.265 & 0.216 & 0.186 & 0.159 & .440 & 0.240 & 0.188 & 0.146 & 0.105 & 0.081 \\
\hline $100 \times 2$ & 74 & 0.053 & 048 & .043 & 37 & 34 & 031 & 0.010 & .006 & 0.004 & 0.002 & 0.001 \\
\hline $100 \times 5$ & 86 & 198 & 190 & 0.170 & 150 & & 180 & 0.108 & .082 & 0.060 & 0.039 & .025 \\
\hline $100 \times 10$ & 406 & 0.304 & 0.279 & 0.241 & 215 & 02 & 311 & 0.182 & 0.136 & 0.103 & 0.065 & 0.042 \\
\hline $100 \times 20$ & 497 & 0.316 & 0.277 & 0.245 & 0.212 & 0.190 & 436 & 0.265 & 0.208 & 0.155 & 0.101 & 0.074 \\
\hline $200 \times 2$ & 44 & 0.037 & 0.035 & 0.033 & 30 & 9 & 022 & 0.008 & 0.006 & 0.004 & 0.002 & 0.002 \\
\hline $200 \times 5$ & 51 & 0.173 & 59 & 46 & & & 36 & 0.081 & 059 & .043 & 026 & .015 \\
\hline $200 \times 10$ & 422 & 0.247 & 0.221 & 0.204 & 90 & & 232 & 0.142 & 0.109 & 0.076 & 0.046 & 0.024 \\
\hline $200 \times 20$ & 583 & 0.283 & 0.240 & 0.213 & 0.200 & 0.189 & 356 & 0.223 & 0.177 & 0.127 & 0.075 & 0.040 \\
\hline \multirow[t]{2}{*}{ Avg } & 29 & 0.202 & 177 & 0.156 & 138 & 26 & 226 & 0.129 & 0.099 & 0.073 & 0.047 & 0.031 \\
\hline & \multicolumn{6}{|c|}{$\overline{\text { PSA-SD }}$} & \multicolumn{6}{|c|}{ PSA-TWPT } \\
\hline Ins. & 10 & 50 & 100 & 200 & 500 & 1000 & 10 & 50 & 100 & 200 & 500 & 1000 \\
\hline $50 \times 2$ & 0.024 & 0.007 & 0.004 & 0.003 & 0.001 & 0.000 & 0.027 & 0.007 & 0.004 & 0.002 & 0.001 & 0.001 \\
\hline $50 \times 5$ & 186 & 0.087 & 0.066 & 0.049 & 0.029 & 00 & 0.179 & 0.086 & 0.064 & 0.042 & 0.026 & 0.016 \\
\hline $50 \times 10$ & 766 & 0.220 & 0.168 & 0.126 & 86 & 34 & .354 & 0.188 & 0.149 & 0.117 & 0.075 & .054 \\
\hline $50 \times 20$ & 446 & 0.254 & 0.200 & 0.149 & 0.103 & & .442 & 0.258 & 0.204 & 0.162 & 0.117 & 0.090 \\
\hline $100 \times 2$ & 030 & 0.010 & 0.006 & 0.004 & 0.002 & 0.001 & 0.031 & 0.010 & 0.007 & 0.004 & 0.002 & 0.001 \\
\hline $100 \times 5$ & 188 & 0.108 & 0.080 & 0.058 & 0.036 & 0.023 & .183 & 0.104 & 0.080 & 0.060 & 0.036 & 0.023 \\
\hline $100 \times 10$ & 307 & 0.177 & 0.141 & 0.104 & 0.066 & 4 & .308 & 0.182 & 0.134 & 0.103 & 0.063 & 0.043 \\
\hline $100 \times 20$ & .427 & 0.259 & 0.199 & 0.153 & 0.105 & 0.074 & 0.435 & 0.272 & 0.213 & 0.169 & 0.110 & 0.077 \\
\hline$\times 2$ & 022 & 0.008 & 0.006 & 0.004 & 0.002 & 0.002 & 0.022 & 0.008 & 0.006 & 0.004 & 0.003 & 0.002 \\
\hline $200 \times 5$ & 138 & 0.079 & 0.060 & 0.043 & 0.026 & 0.015 & 0.138 & 0.082 & 0.061 & 0.045 & 0.026 & 0.015 \\
\hline $200 \times 10$ & 229 & 0.143 & 0.111 & 0.083 & & & 0.234 & 0.144 & 0.109 & 0.080 & 0.047 & 0.026 \\
\hline $200 \times 20$ & 0.352 & 0.223 & 0.163 & 0.122 & 0.069 & 0.034 & 0.355 & 0.214 & 0.166 & 0.121 & 0.070 & 0.038 \\
\hline Avg & 0.227 & 0.131 & 0.100 & 0.075 & 0.048 & 0.032 & 0.226 & 0.130 & 0.100 & 0.076 & 0.048 & 0.032 \\
\hline
\end{tabular}

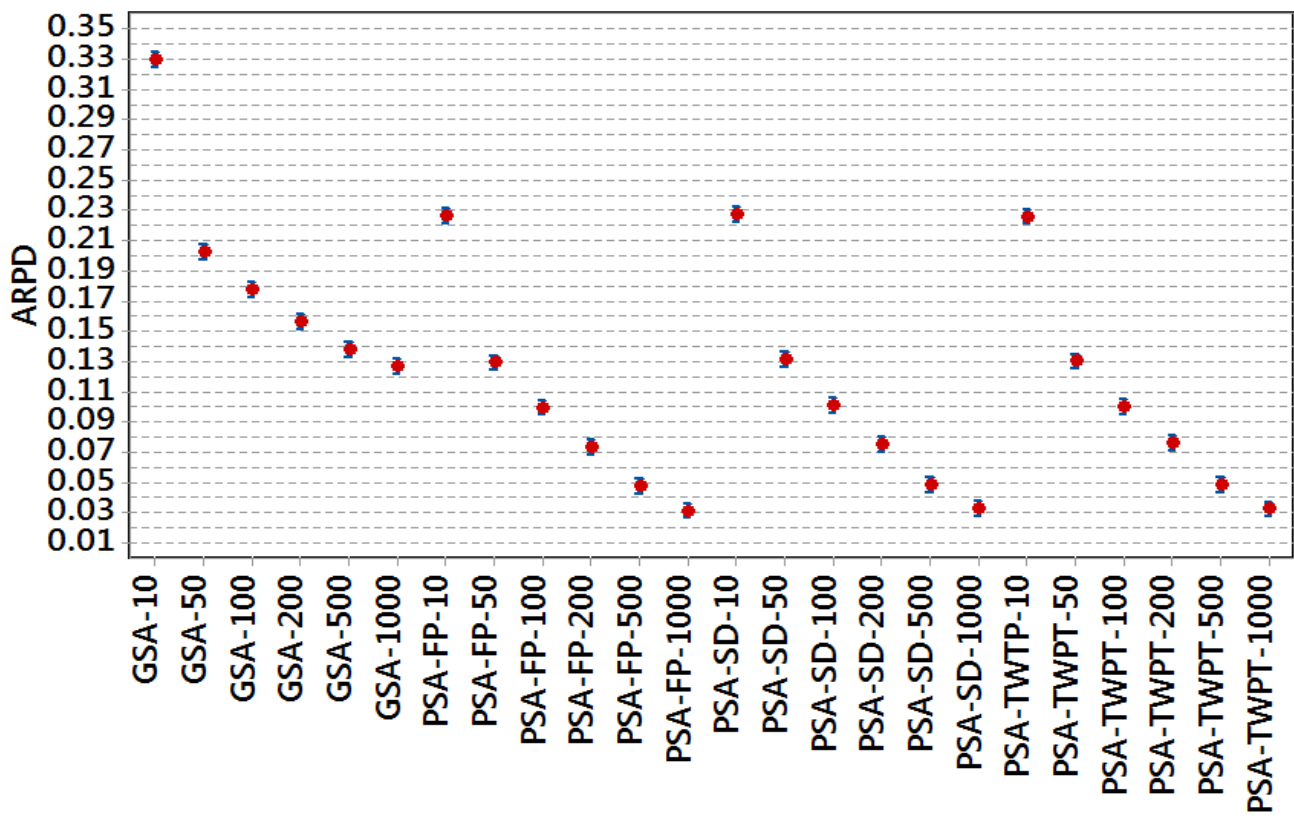

Figure 10: 95\% confidence interval plot of the algorithms compared on Test-1.

although our results showed that our PSA does not depend much on the quality of initial solution. From these graphs, we see the convergence speed of GSA is faster than that of PSA. Convergence graphs for other instances are similar and hence not shown. 
Table 4: ARPDs for the search algorithms on Test-2

\begin{tabular}{|c|c|c|c|c|c|c|c|c|c|c|c|c|}
\hline & \multicolumn{6}{|c|}{ GSA } & \multicolumn{6}{|c|}{ PSA-FP } \\
\hline Ins. & 10 & 50 & 100 & 200 & 500 & 1000 & 10 & 50 & 100 & 200 & 500 & 1000 \\
\hline $50 \times 2$ & 0.106 & 0.052 & 0.037 & 0.029 & 0.019 & 0.014 & 0.033 & 0.009 & 0.004 & 0.002 & 0.001 & 0.000 \\
\hline $50 \times 5$ & 0.439 & 0.242 & 0.201 & 0.160 & 0.128 & 0.103 & 0.259 & 0.123 & 0.085 & 0.063 & 0.039 & 0.028 \\
\hline $50 \times 10$ & 0.623 & 0.322 & 0.257 & 0.211 & 0.167 & 0.143 & 0.369 & 0.173 & 0.131 & 0.097 & 0.060 & 0.040 \\
\hline $50 \times 20$ & 0.689 & 0.297 & 0.235 & 0.208 & 0.149 & 0.135 & 0.427 & 0.226 & 0.170 & 0.127 & 0.082 & 0.047 \\
\hline $100 \times 2$ & 0.096 & 0.056 & 0.051 & 0.040 & 0.035 & 0.031 & 0.028 & 0.010 & 0.007 & 0.004 & 0.002 & 0.001 \\
\hline $100 \times 5$ & 0.408 & 0.294 & 0.286 & 0.251 & 0.219 & 0.209 & 0.258 & 0.142 & 0.109 & 0.079 & 0.047 & 0.029 \\
\hline $100 \times 10$ & 0.557 & 0.382 & 0.352 & 0.315 & 0.270 & 0.255 & 0.437 & 0.254 & 0.185 & 0.135 & 0.083 & 0.052 \\
\hline $100 \times 20$ & 0.588 & 0.382 & 0.354 & 0.325 & 0.272 & 0.258 & 0.589 & 0.349 & 0.264 & 0.206 & 0.126 & 0.085 \\
\hline $200 \times 2$ & 0.051 & 0.044 & 0.040 & 0.036 & 0.032 & 0.030 & 0.020 & 0.007 & 0.004 & 0.003 & 0.002 & 0.001 \\
\hline $200 \times 5$ & 0.289 & 0.228 & 0.218 & 0.209 & 0.190 & 0.178 & 0.208 & 0.116 & 0.085 & 0.061 & 0.036 & 0.022 \\
\hline $200 \times 10$ & 0.475 & 0.344 & 0.321 & 0.297 & 0.276 & 0.261 & 0.402 & 0.235 & 0.183 & 0.133 & 0.077 & 0.044 \\
\hline $200 \times 20$ & 0.554 & 0.365 & 0.312 & 0.275 & 0.247 & 0.234 & 0.548 & 0.363 & 0.288 & 0.219 & 0.121 & 0.071 \\
\hline \multirow[t]{2}{*}{ Avg } & 0.406 & 0.251 & 0.222 & 0.196 & 0.167 & 0.154 & 0.298 & 0.167 & 0.126 & 0.094 & 0.056 & 0.035 \\
\hline & \multicolumn{6}{|c|}{$\overline{\mathrm{PSA}-\mathrm{SD}}$} & \multicolumn{6}{|c|}{ 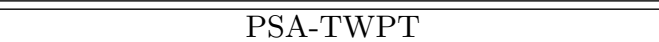 } \\
\hline Ins. & 10 & 50 & 100 & 200 & 500 & 1000 & 10 & 50 & 100 & 200 & 500 & 1000 \\
\hline $50 \times 2$ & 0.031 & 0.008 & 0.004 & 0.002 & 0.001 & 0.000 & 0.033 & 0.008 & 0.005 & 0.002 & 0.001 & 0.000 \\
\hline $50 \times 5$ & 0.263 & 0.122 & 0.094 & 0.061 & 0.037 & 0.028 & 0.256 & 0.129 & 0.097 & 0.063 & 0.037 & 0.025 \\
\hline $50 \times 10$ & 0.391 & 0.193 & 0.137 & 0.093 & 0.054 & 0.036 & 0.397 & 0.200 & 0.141 & 0.095 & 0.060 & 0.041 \\
\hline $50 \times 20$ & 0.413 & 0.224 & 0.162 & 0.113 & 0.078 & 0.060 & 0.424 & 0.200 & 0.148 & 0.107 & 0.061 & 0.047 \\
\hline $100 \times 2$ & 0.027 & 0.010 & 0.006 & 0.003 & 0.002 & 0.001 & 0.029 & 0.009 & 0.005 & 0.003 & 0.002 & 0.001 \\
\hline $100 \times 5$ & 0.260 & 0.142 & 0.106 & 0.077 & 0.046 & 0.027 & 0.257 & 0.143 & 0.103 & 0.078 & 0.047 & 0.030 \\
\hline $100 \times 10$ & 0.434 & 0.243 & 0.187 & 0.136 & 0.081 & 0.054 & 0.433 & 0.251 & 0.184 & 0.138 & 0.094 & 0.059 \\
\hline $100 \times 20$ & 0.595 & 0.353 & 0.272 & 0.207 & 0.131 & 0.089 & 0.571 & 0.341 & 0.268 & 0.201 & 0.125 & 0.084 \\
\hline $200 \times 2$ & 0.021 & 0.008 & 0.005 & 0.003 & 0.002 & 0.001 & 0.020 & 0.007 & 0.005 & 0.003 & 0.002 & 0.001 \\
\hline $200 \times 5$ & 0.211 & 0.112 & 0.081 & 0.061 & 0.037 & 0.020 & 0.215 & 0.116 & 0.083 & 0.060 & 0.035 & 0.019 \\
\hline $200 \times 10$ & 0.406 & 0.248 & 0.193 & 0.139 & 0.076 & 0.042 & 0.407 & 0.247 & 0.185 & 0.132 & 0.074 & 0.041 \\
\hline $200 \times 20$ & 0.559 & 0.371 & 0.290 & 0.211 & 0.121 & 0.066 & 0.551 & 0.366 & 0.289 & 0.211 & 0.124 & 0.066 \\
\hline Avg & 0.301 & 0.170 & 0.128 & 0.092 & 0.055 & 0.035 & 0.299 & 0.168 & 0.126 & 0.091 & 0.055 & 0.035 \\
\hline
\end{tabular}

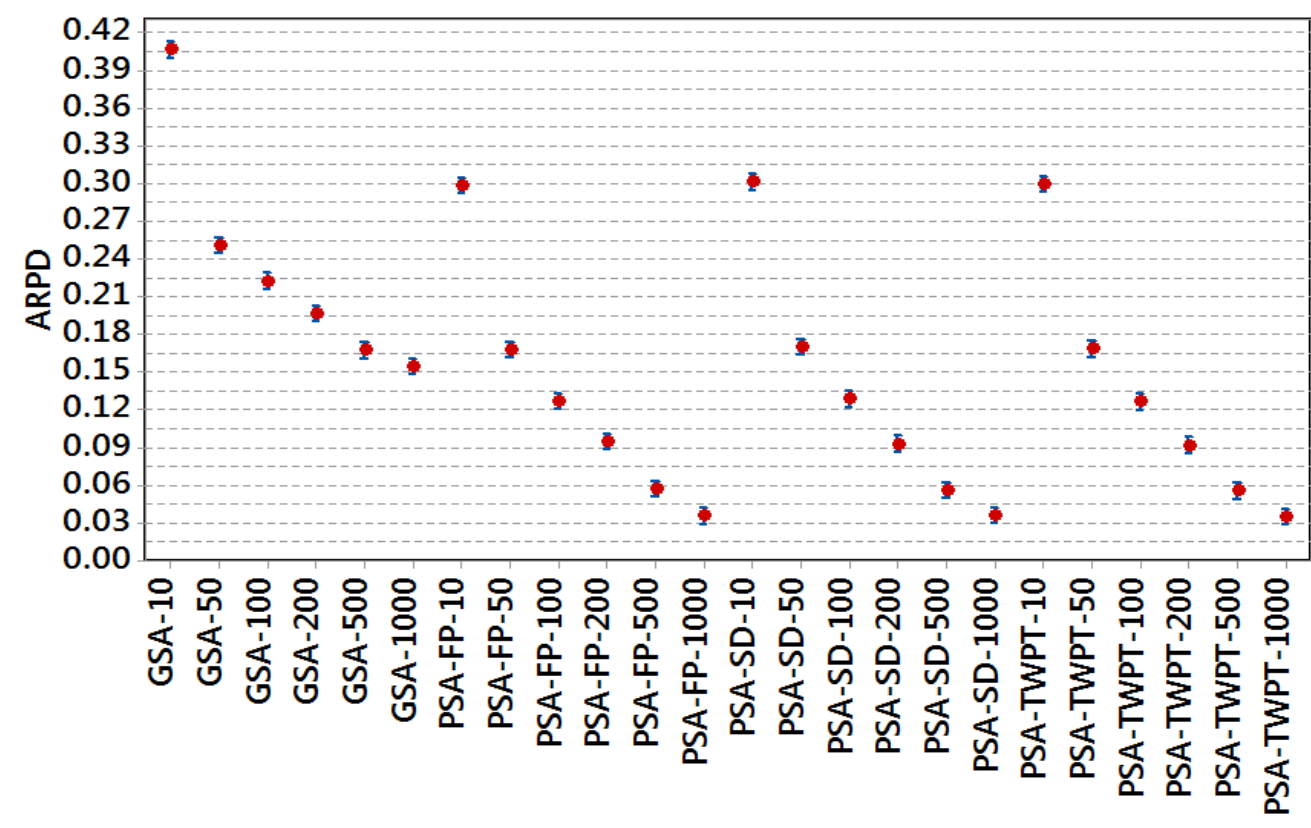

Figure 11: 95\% confidence interval plot of the algorithms compared on Test-2.

Since the search process of the PSA (using FP heuristic as initial point) and GSA in each iteration is not the same, we also report the CPU times of those two algorithms in Table 5. Moreover, we report the relative deviation $R D=100 \times(G S A-P S A) / P S A$ of CPU time. As 

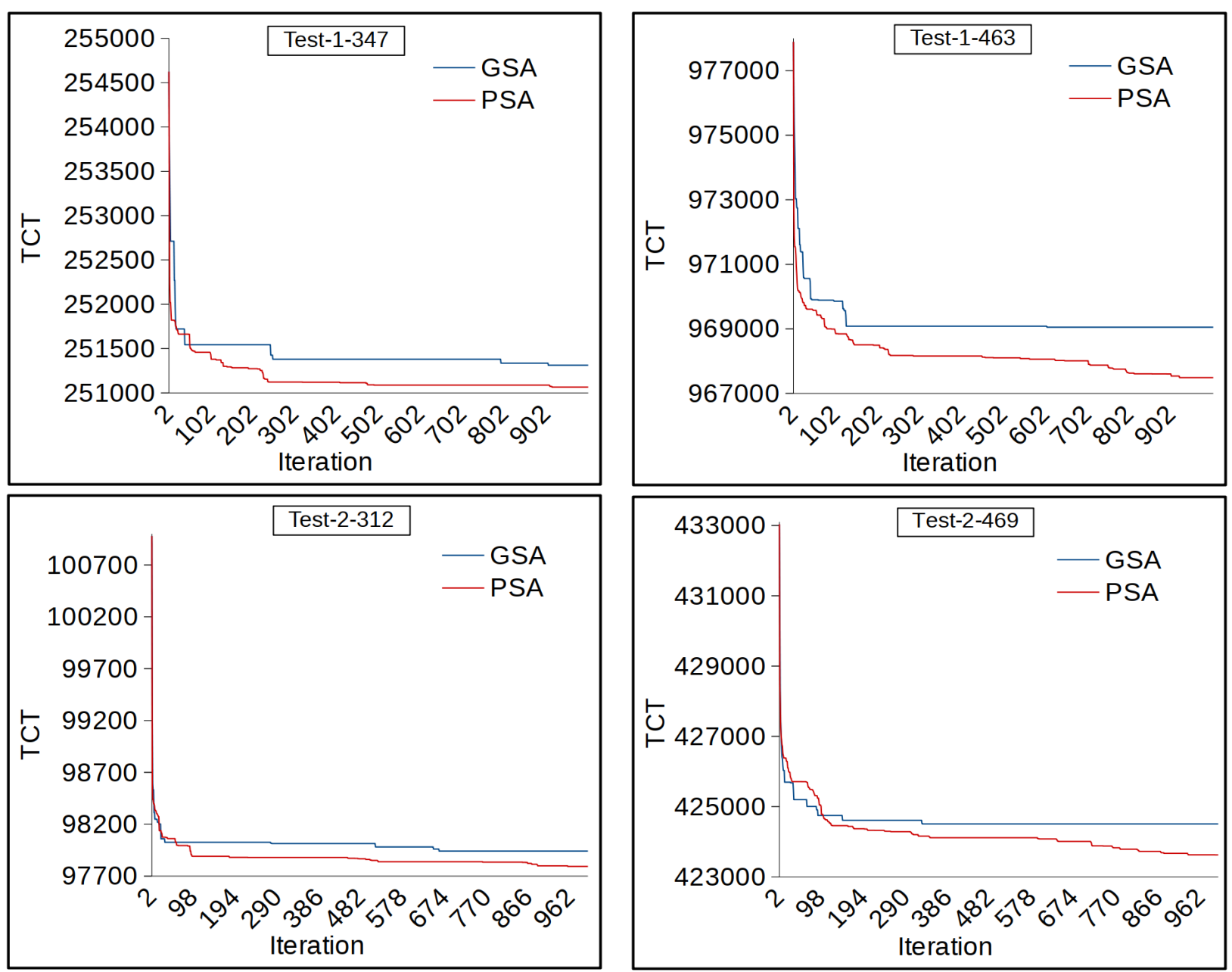

Figure 12: Convergence curves of GSA and PSA algorithms on four selected instances.

can be seen, the PSA algorithm is much faster than GSA, $11 \%$ in overall average, yet finds better solutions as shown in Tables 3 and 4 .

Table 5: CPU times needed for GSA and PSA algorithms.

\begin{tabular}{c|rr|r}
\hline Instance & GSA & PSA & RD \\
\hline $50 \times 2$ & 1.99 & 1.74 & 14.44 \\
$50 \times 5$ & 3.59 & 3.51 & 2.07 \\
$50 \times 10$ & 6.48 & 6.35 & 2.13 \\
$50 \times 20$ & 12.16 & 11.65 & 4.33 \\
$100 \times 2$ & 11.80 & 11.34 & 4.05 \\
$100 \times 5$ & 26.65 & 23.94 & 11.30 \\
$100 \times 10$ & 48.74 & 43.88 & 11.07 \\
$100 \times 20$ & 92.05 & 82.91 & 11.01 \\
$200 \times 2$ & 89.79 & 80.70 & 11.27 \\
$200 \times 5$ & 208.20 & 175.79 & 18.44 \\
$200 \times 10$ & 386.46 & 321.24 & 20.30 \\
$200 \times 20$ & 765.25 & 609.57 & 25.54 \\
\hline average & 137.76 & 114.39 & 11.33 \\
\hline
\end{tabular}

Finally, the best found solutions for the instances of Framinan and Perez-Gonzalez (2017) obtained by any of the comparing algorithms are reported in the online materials. These best 
found solutions are already used in this paper in the ARPD calculation. Table 6 summarises the key results. As can be seen, almost all of the best found solutions are obtained by the proposed PSA algorithms.

Table 6: The number of best found solutions obtained by the four algorithms

\begin{tabular}{ccccc}
\hline Instances & \multicolumn{4}{c}{ Algorithms } \\
\hline Total 720 & GSA & PSA-FP & PSA-SD & PSA \\
Test-1 & 3 & $\mathbf{1 7 7}$ & 173 & 153 \\
Test-2 & 8 & 201 & 197 & $\mathbf{2 0 2}$ \\
\hline Total & 11 & $\mathbf{3 7 8}$ & 370 & 355 \\
\hline
\end{tabular}

\section{Conclusion}

In this paper, we study the Customer Order Scheduling Problem (COSP) with TCT minimisation, which is known to be NP-Hard. The COSP has realistic applications that include the paper and the pharmaceutical industries. In this paper, we propose constructive and perturbative search algorithms for COSP embedding problem specific heuristics within. We propose effective dispatching rules to be used during solution construction. We propose greedy ways to select customer orders that should be rescheduled during the diversification phase of a perturbative search algorithm. We also diversify the intensification phase of the perturbative search algorithm by using multiple neighbourhood operators. Our experimental results show that the proposed algorithms outperform existing state-of-the-art COSP algorithms. Moreover, our proposed algorithms have found the best solutions in almost all benchmark problem instances used in the experiments.

\section{Acknowledgement}

The authors sincerely appreciate Professor Jose M. Framinan as he shared GSA and FP implementations.

\section{References}

Ahmadi, R., Bagchi, U., and Roemer, T. A. (2005). Coordinated scheduling of customer orders for quick response. Naval Research Logistics (NRL), 52(6):493-512.

Framinan, J. M. and Perez-Gonzalez, P. (2017). New approximate algorithms for the customer order scheduling problem with total completion time objective. Computers 85 Operations Research, 78:181-192.

Framinan, J. M. and Perez-Gonzalez, P. (2018). Order scheduling with tardiness objective: Improved approximate solutions. European Journal of Operational Research, 266(3):840-850.

Framinan, J. M., Perez-Gonzalez, P., and Fernandez-Viagas, V. (2018). Deterministic assembly scheduling problems: A review and classification of concurrent-type scheduling models and solution procedures. European Journal of Operational Research, 273:401-417.

Leung, J. Y., Li, H., and Pinedo, M. (2005a). Order scheduling models: an overview. In Multidisciplinary scheduling: theory and applications, pages 37-53. Springer.

Leung, J. Y.-T., Li, H., and Pinedo, M. (2005b). Order scheduling in an environment with dedicated resources in parallel. Journal of Scheduling, 8(5):355-386.

Lin, B. M. and Kononov, A. V. (2007). Customer order scheduling to minimize the number of late jobs. European Journal of Operational Research, 183(2):944-948.

Lin, W.-C., Yin, Y., Cheng, S.-R., Cheng, T. E., Wu, C.-H., and Wu, C.-C. (2017). Particle swarm optimization and opposite-based particle swarm optimization for two-agent multi-facility customer order scheduling with ready times. Applied Soft Computing, 52:877-884.

Montgomery, D. C. (2017). Design and analysis of experiments. John Wiley \& Sons.

Riahi, V., Newton, M. H., Su, K., and Sattar, A. (2018). Local search for flowshops with setup times and blocking constraints. In International Conference on Automated Planning and Scheduling (ICAPS), pages 199-207.

Roemer, T. and Ahmadi, R. (1997). The complexity of scheduling customer orders. In Proceedings of the informs conference.

Shi, Z., Huang, Z., and Shi, L. (2018). Customer order scheduling on batch processing machines with incompatible job families. International Journal of Production Research, 56(1-2):795-808. 
Shi, Z., Wang, L., Liu, P., and Shi, L. (2017). Minimizing completion time for order scheduling: formulation and heuristic algorithm. IEEE Transactions on Automation Science and Engineering, 14(4):1558-1569.

Sung, C. S. and Yoon, S. H. (1998). Minimizing total weighted completion time at a pre-assembly stage composed of two feeding machines. International Journal of Production Economics, 54(3):247-255.

Wu, C.-C., Liu, S.-C., Lin, T.-Y., Yang, T.-H., Chung, I.-H., and Lin, W.-C. (2018). Bicriterion total flowtime and maximum tardiness minimization for an order scheduling problem. Computers $\mathcal{G}$ Industrial Engineering, 117:152-163.

Xu, J., Wu, C.-C., Yin, Y., Zhao, C., Chiou, Y.-T., and Lin, W.-C. (2016a). An order scheduling problem with position-based learning effect. Computers 83 Operations Research, 74:175-186.

Xu, X., Ma, Y., Zhou, Z., and Zhao, Y. (2015). Customer order scheduling on unrelated parallel machines to minimize total completion time. IEEE Transactions on Automation Science and Engineering, 12(1):244-257.

Xu, X., Zhao, Y., Wu, M., Zhou, Z., Ma, Y., and Liu, Y. (2016b). Stochastic customer order scheduling to minimize long-run expected order cycle time. Annals of Operations Research, pages 1-24.

Yang, J. (1998). Scheduling with batch objectives. PhD thesis, The Ohio State University. 